\title{
Monitoring Alzheimer Amyloid Peptide Aggregation by EPR
}

\author{
I. Sepkhanova $\cdot$ M. Drescher $\cdot$ N. J. Meeuwenoord $\cdot$ \\ R. W. A. L. Limpens • R. I. Koning • D. V. Filippov • \\ M. Huber
}

Received: 30 November 2008/Revised: 28 December 2008/Published online: 31 October 2009

(C) The Author(s) 2009. This article is published with open access at Springerlink.com

\begin{abstract}
Plaques containing the aggregated $\beta$-Amyloid $(\mathrm{A} \beta)$ peptide in the brain are the main indicators of Alzheimer's disease. Fibrils, the building blocks of plaques, can also be produced in vitro and consist of a regular arrangement of the peptide. The initial steps of fibril formation are not well understood and could involve smaller aggregates (oligomers) of $\mathrm{A} \beta$. Such oligomers have even been implicated as the toxic agents. Here, a method to study oligomers on the time scale of aggregation is suggested. We have labeled the 40 residue $\mathrm{A} \beta$ peptide variant containing an N-terminal cysteine (cys-A $\beta$ ) with the MTSL [1-oxyl-2,2,5,5-tetramethyl- $\Delta$-pyrroline-3-methyl] methanethiosulfonate spin label (SL-A $\beta$ ). Fibril formation in solutions of pure SL-A $\beta$ and of SL-A $\beta$ mixed with $\mathrm{A} \beta$ was shown by Congo-red binding and electron microscopy. Continuous-wave $9 \mathrm{GHz}$ electron paramagnetic resonance reveals three fractions of different spin-label mobility: one attributed to monomeric $\mathrm{A} \beta$, one to a multimer (8-15 monomers), and the last one to larger aggregates or fibrils. The approach, in principle, allows detection of oligomers on the time scale of aggregation.
\end{abstract}

I. Sepkhanova $\cdot$ M. Drescher $\cdot$ M. Huber $(\bowtie)$

Huygens Laboratory, Department of Molecular Physics, Leiden University,

P.O. Box 9504, 2300 RA Leiden, The Netherlands

e-mail: mhuber@molphys.leidenuniv.nl

N. J. Meeuwenoord · D. V. Filippov

Leiden Institute of Chemistry, Leiden University, Leiden, The Netherlands

R. W. A. L. Limpens · R. I. Koning

Section Electron Microscopy, Department of Molecular Cell Biology,

Leiden University Medical Center, Leiden, The Netherlands 


\section{Introduction}

The aggregation of the $\beta$-Amyloid $(\mathrm{A} \beta)$ peptide into fibrils and ultimately plaques is the chief indicator of Alzheimer's disease. Information on the structure of the fibrils had been obtained from microscopy techniques such as cryo-electron microscopy (cryo-EM) [1], atomic force microscopy (AFM), scanning tunneling microscopy (STM) [2], solid-state nuclear magnetic resonance (ss-NMR) [3-5] and liquid solution NMR [6] methods. The fibrils of $A \beta$ and other amylogenic proteins, such as $\alpha$-synuclein, have been studied by spin-label electron paramagnetic resonance (EPR), targeting the mobility of the spin-label and spin-spin interaction [7-9]. Even X-ray crystallography was possible on nanocrystals grown from a short peptide model [10].

The aggregation process itself, particularly the role of oligomers in the aggregation, is still much less understood. Specific interest in such smaller aggregates stems from the suggestion that small, oligomeric aggregates and protofibrils, rather than fully formed fibrils could be responsible for the toxicity of the $\mathrm{A} \beta$-peptide. Studies targeting oligomers of $\mathrm{A} \beta$ via diffusion measurements by NMR [11], size-exclusion chromatography [12], infrared spectroscopy [13] and mass spectrometry [14] have been published.

In the present study, we investigate the potential of EPR to detect early stages of the aggregation of the $\mathrm{A} \beta$-peptide. Whereas previous EPR was performed on fibrils separated from the aggregating solution after a longer incubation time [7-9], the present investigation focuses on the reaction mixture to study the process of aggregation itself. We employ the spin-labeled cysteine mutant SL-A $\beta$ and the unlabeled $\mathrm{A} \beta$ (wild-type $\mathrm{A} \beta 1-40$ ) to diamagnetically dilute the spins [7, 8]. Earlier, EPR signatures of aggregation in a prion protein were described. These signatures were assigned to aggregates large enough to be immobilized on the EPR time scale [15]. Recently, an abstract about EPR on $\mathrm{A} \beta(1-25)$ [16] has been published. We demonstrate that signatures of oligomers can be detected by our methodology and that different species coexist in the aggregating solution. The approach should make it possible to follow the time scale of the aggregation.

\section{Materials and Methods}

The peptide $\mathrm{A} \beta-(1-40)$ as well as its cysteine mutant Cys-(A $\beta)$ (H-Cys-Asp-Ala--..Val-OH) was purchased from AnaSpec (purity $>95 \%$ ), the solvent dimethyl sulfoxide (DMSO) was purchased from Biosolve (purity 99.8\%), spin probes MTSL [1-oxyl-2,2,5,5-tetramethyl- $\Delta$-pyrroline-3-methyl] methanethiosulfonate were purchased from Toronto Research Chemicals Inc. (Brisbane Rd., NorthYork, ON, Canada, M3J 2J8) and were used without further purification. Phosphate-buffered saline (PBS, $12 \mathrm{mM} \mathrm{Na}_{2} \mathrm{HPO}_{4}, 137 \mathrm{mM} \mathrm{NaCl}, 2.7 \mathrm{mM} \mathrm{KCl}, 1.8 \mathrm{mM} \mathrm{KH}_{2} \mathrm{PO}_{4}$ at $\mathrm{pH}$ 7.4) was used to initiate aggregation of $\mathrm{A} \beta$.

\subsection{Spin Labeling}

Cys-(A $\beta)$ was dissolved in DMSO $(2.5 \mathrm{mg} / \mathrm{ml})$, Tris-HCl buffer $(10 \mathrm{mM}, \mathrm{pH} 8.8$, sonicated under vacuum) was added to a final peptide concentration of $0.33 \mathrm{mg} / \mathrm{ml}$. 
After adding a fivefold molar excess of MTSL spin label $\left(M_{\mathrm{W}}=264.3 \mathrm{~g} / \mathrm{mol}\right)$, the solution was incubated under shaking for $60 \mathrm{~min}$.

Spin-labeled Cys- $(\mathrm{A} \beta)(\mathrm{SL}-\mathrm{A} \beta)$ was purified on a semi-preparative Gemini ${ }^{\circledR} \mathrm{C} 18$ column (Phenomenex ${ }^{\circledR}$ ), $250 \mathrm{~mm} \times 10 \mathrm{~mm}, 5 \mu \mathrm{m}$ particle size, by applying a gradient of $\mathrm{B}$ in $\mathrm{A}, \mathrm{C}$ kept isocratic at $10 \%$ and by increasing the amount of $\mathrm{B}$ from 10 to $70 \%$ [A, $\mathrm{H}_{2} \mathrm{O}$; B, acetonitrile; $\mathrm{C}, \mathrm{H}_{2} \mathrm{O} 1 \mathrm{vol} \%$ aqueous trifluoroacetic acid (TFA)]. The purified SL-A $\beta$ was analyzed by liquid chromatography-mass spectrometry. The analytical high performance liquid chromatography column was Gemini ${ }^{\circledR} \mathrm{C} 18\left(\right.$ Phenomenex $^{\circledR}$ ), $50 \mathrm{~mm} \times 4.6 \mathrm{~mm}, 3 \mu \mathrm{m}$ particle size, applying a gradient of B in A (10-90\% B), C kept isocratic at $10 \%\left(\mathrm{~A}, \mathrm{H}_{2} \mathrm{O}\right.$; B acetonitrile; C, $\mathrm{H}_{2} \mathrm{O} 1$ vol\% aqueous TFA). The mass spectrometer was a PE Sciex API 165 single quadrupole instrument. Found: $m / z=1540.13$ for $(M+3 \mathrm{H})^{3+}$ (calculated for $100 \%$ abundance, 1539.76) and $m / z=1155.27$ for $(M+4 \mathrm{H})^{4+}$ (calculated for $100 \%$ abundance, 1155.07). Retention time, $t_{\mathrm{r}}=5.69 \mathrm{~min}$. The peptide was lyophilized and stored in the freezer $\left(-20^{\circ} \mathrm{C}\right)$ until used.

\subsection{Samples for EPR Spectroscopy, Room Temperature EPR}

Samples of SL-A $\beta$ in DMSO were prepared as a reference with a peptide concentration of $0.22 \mathrm{mM}$. The SL-A $\beta$ samples in PBS were prepared as diamagnetically diluted samples (dd-SL-A $\beta$ ), containing a molar fraction of 14 or $40 \%$ labeled peptide. The total peptide concentration was kept constant at $0.55 \mathrm{mM}$ for all room temperature EPR measurements containing $76 \mu \mathrm{M}$ and $0.22 \mathrm{mM}$ SL-A $\beta$ for the 14 and $40 \%$ labeled samples, respectively. Samples have been diluted in an aqueous PBS solution to trigger aggregation. The concentration of DMSO in such samples has been kept below 10\%. For room temperature measurements, samples of 10-15 $\mu \mathrm{l}$ peptide solution were drawn into Blaubrand $50 \mu$ l capillaries. Often, a white precipitate was observed. Each experiment shown was repeated at least twice. In total, more than 30 samples were investigated and methods were varied in a limited range to improve sample preparation and aggregation conditions. The database is not sufficient to systematically study sample-to-sample variations yet. The measurements were made immediately after dissolving in PBS. The samples have been incubated at room temperature in these capillaries as well as in vials under shaking $(800 \mathrm{rpm})$ for 9 days and measured again to monitor timedependent effects. Beyond this, no attempts were made to vary the aggregation protocol as described in refs. [17-21].

\subsection{Filtration Experiment}

A $40 \%$ SL-A $\beta$ sample with a total concentration of $0.55 \mathrm{mM}$ protein was dissolved in PBS and was left to incubate for $40 \mathrm{~min}$. Subsequently, it was filtered using a $30 \mathrm{kDa}$ membrane filter (Vivaspin 500, Vivascience, Satorius). Three hours after starting the incubation, a sample of the initial solution, the retentate and the filtrate were measured. The filtrate was measured as obtained, and $150 \mu$ of PBS was added to the retentate (ca. $25 \mu \mathrm{l}$ ) just before measuring. 


\subsection{Spin Concentration}

The spin concentration in the samples in PBS was determined relative to that of the DMSO stock solution of SL-A $\beta$ by double integration of the first-derivative EPR spectra. The errors of this method are around $15 \%$ due to difficulties with the baseline subtraction in the spectra.

\subsection{Frozen-Solution EPR}

For measurements in frozen solution, a droplet of SL-A $\beta$ stock solution was diluted in the EPR tube (4 mm o.d.) to a concentration of $80 \mu \mathrm{M}$ in PBS or DMSO, respectively. After incubation for $5 \mathrm{~min}, 20 \%$ (v/v) glycerol has been added. Measurements were performed at $120 \mathrm{~K}$ after shock freezing the sample in liquid nitrogen.

\subsection{EPR Spectrometer}

The X-band continuous-wave (cw) EPR measurements were performed on an Elexsys E 680 spectrometer (Bruker, Rheinstetten, Germany) equipped with a helium gas-flow cryostat and a rectangular cavity. A modulation frequency of $100 \mathrm{kHz}$ was used for all measurements; the accumulation time for the spectra shown and for those used for simulation of the line shape was 40 min per spectrum. Information on the time course of aggregation is contained in the ratio of the fast, intermediate and slow components. It can be obtained from spectra taken in 5-10 min range, making use of the line shape determined for spectra with longer accumulation time. Measurements in liquid solution at room temperature were done at $6.31 \mathrm{~mW}$ microwave power and modulation amplitudes of 0.25 and $1.4 \mathrm{G}$. The larger modulation amplitude was used to obtain a better signal-to-noise ratio of broad lines. The sample height was carefully adjusted in order to be sensitive to that part of the solution, where white precipitates have been observed. For measurements in frozen solution at $120 \mathrm{~K}$, a modulation amplitude of $2 \mathrm{G}$ and microwave power of $0.05 \mathrm{~mW}$ have been used. Spin concentrations were compared via the double integral of the spectra of $\mathrm{A} \beta$ in DMSO and in PBS.

\subsection{Simulations of EPR Spectra}

The spectra were simulated using Matlab and the EasySpin [22] package. For room temperature spectra, up to three spectral components featuring different degrees of rotational mobility of the spin labels have been taken into account. For these spectral contributions, the following parameters were used: $g=[2.009006$, 2.00687, 2.003] [23], and $A_{\mathrm{xx}}=A_{\mathrm{yy}}=12$ and $13 \mathrm{MHz}$ in DMSO and PBS, respectively. Simulating the spectrum of SL-A $\beta$ in DMSO and of the fast component of the PBS samples, a hyperfine interaction with six carbon sites was taken into account assuming a natural abundance of ${ }^{13} \mathrm{C}$ of $1 \%$. An isotropic hyperfine splitting $A_{13 \mathrm{C}}=17 \mathrm{MHz}$ was used. Over-modulation effects were taken into account if appropriate. 
The simulations for both normal and over-modulated spectra are possible with the EasySpin program and all parameters for narrow lines are the same when the function for an over-modulated spectrum is used (all spectra shown are overmodulated spectra).

For the low-temperature spectra, the same $g$ tensor as for room temperature was used with $A_{\mathrm{xx}}=A_{\mathrm{yy}}=12 \mathrm{MHz}$ for both DMSO and PBS.

\subsection{Interpretation of Rotation Correlation Times}

Rotation correlation times were interpreted with the Stokes-Einstein relation

$$
\tau_{\mathrm{R}}=\frac{4 \pi \eta \alpha^{3}}{3 \mathrm{kT}}
$$

A spherical approximation is used for the shape of the aggregates. For the fast rotating fraction of the sample, a $\tau_{\mathrm{R}}$ of ca. $0.15 \mathrm{~ns}$ is obtained. Using a viscosity of water of $\eta=1 \times 10^{-3} \mathrm{~kg} \mathrm{~s} / \mathrm{m}$ at $20^{\circ} \mathrm{C}$, a volume of $606 \AA^{3}$ results, which is close to the volume of $529 \AA^{3}$ obtained from the $\tau_{\mathrm{R}}$ of A $\beta$ in DMSO $(\eta=1.99 \mathrm{~kg} \mathrm{~s} / \mathrm{m}$, $\left.\tau_{R}=0.26 \mathrm{~ns}\right)$. For the fraction with the intermediate $\tau_{R}\left(\tau_{R}=2.3 \mathrm{~ns}\right)$, a volume of $9,304 \AA^{3}$ results.

\subsection{Congo-Red Binding Assay}

Aggregation in EPR samples (dd-SL-A $\beta$ ) has been checked additionally by the Congo-red binding assay. The optical absorbance has been measured in the range of 400-700 nm in cuvettes of $1 \mathrm{ml}$ containing $9.84 \mu \mathrm{M}$ Congo red in PBS before and after adding peptide solution to a final peptide concentration of $8.8 \mu \mathrm{M}$ $[24,25]$.

\subsection{Electron Microscopy}

Negative staining was performed by placing $5 \mu \mathrm{l}$ of the peptide solution on glowdischarged carbon supported by an EM grid. The droplet was allowed to absorb for about $1 \mathrm{~min}$ and then blotted off. The samples were rinsed with a drop of water and negatively stained with one drop of $3 \%$ uranyl acetate. The transmission electron microscopy (TEM) was performed using a $100 \mathrm{kV}$ Phillips CM10 transmission electron microscope and images were recorded using a side-mounted chargecoupled device (CCD) camera (Megaview III, SIS, Olympus).

\section{Results}

In order to verify that fibrils are formed, the solutions of incubated $\mathrm{A} \beta$ were investigated using the Congo-red binding assay and electron microscopy. 
Fig. 1 TEM images of $\mathrm{A} \beta$.

After 5 min (top, scale bar $200 \mathrm{~nm}$ ) and after $15 \mathrm{~min}$ of incubation (bottom same scale as top)
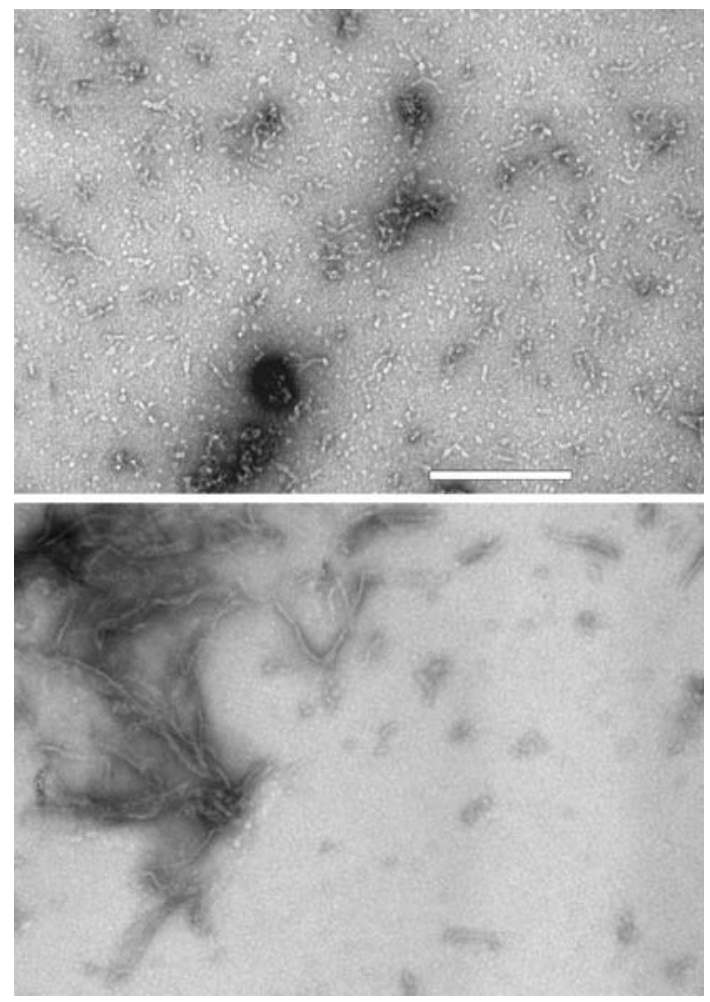

\subsection{Congo-Red Binding and Electron Microscopy}

For the $14 \%$ labeled sample, the characteristic $4 \mathrm{~nm}$ shift of the absorbance at $487 \mathrm{~nm}$, which is expected for fibrils [24, 25], was observed. Electron microscopy was performed on pure SL-A $\beta$, on pure $\mathrm{A} \beta$ and on mixed SL-A $\beta / \mathrm{A} \beta$ samples that had been used for EPR. Figure 1 shows two representative EM pictures of 14\% $\mathrm{SL}-\mathrm{A} \beta$, one at short times (after $5 \mathrm{~min}$ ), and one after $15 \mathrm{~min}$. After $5 \mathrm{~min}$, the majority of the features are very short fibrils with a width of 5-10 $\mathrm{nm}$ and a length of 20-100 nm, in agreement with the dimensions of protofibrils found by EM [26] and AFM [27]. After 15 min, very small fibrils as above and larger fibrils with a width of $5-10 \mathrm{~nm}$ and a length of $100 \mathrm{~nm}-1 \mu \mathrm{m}$ occur. Some of these larger fibrils are stuck together, but are still recognizable as fibrils. Further, some irregularly shaped clusters, presumably large aggregates of peptides, of diameter $200 \mathrm{~nm}-1 \mu \mathrm{m}$, are found. After $60 \mathrm{~min}$, very small fibrils as above, almost no intermediate size fibrils, and more condensed clustered aggregates with a diameter of $200 \mathrm{~nm}-1 \mu \mathrm{m}$ are found. After several days of aggregation, the main structural features were clustered aggregates with a diameter of $200 \mathrm{~nm}-1 \mu \mathrm{m}$. Samples on which EPR had been measured previously also showed fibril signatures as described above. 
Fig. 2 Room temperature EPR spectra of SL-A $\beta$. a SL-A $\beta$ in DMSO: solid line experiment, dotted line simulation. b $40 \%$ $\mathrm{SL}-\mathrm{A} \beta$ in PBS after $5 \mathrm{~min}$ of incubation: solid line experiment, dotted line simulation. c Spectrum of $40 \%$ $\mathrm{SL}-\mathrm{A} \beta$ in PBS after $10 \mathrm{~min}$ incubation and simulation of the spectrum with three components: dotted light gray line fast, dark gray line medium, solid gray line slow component

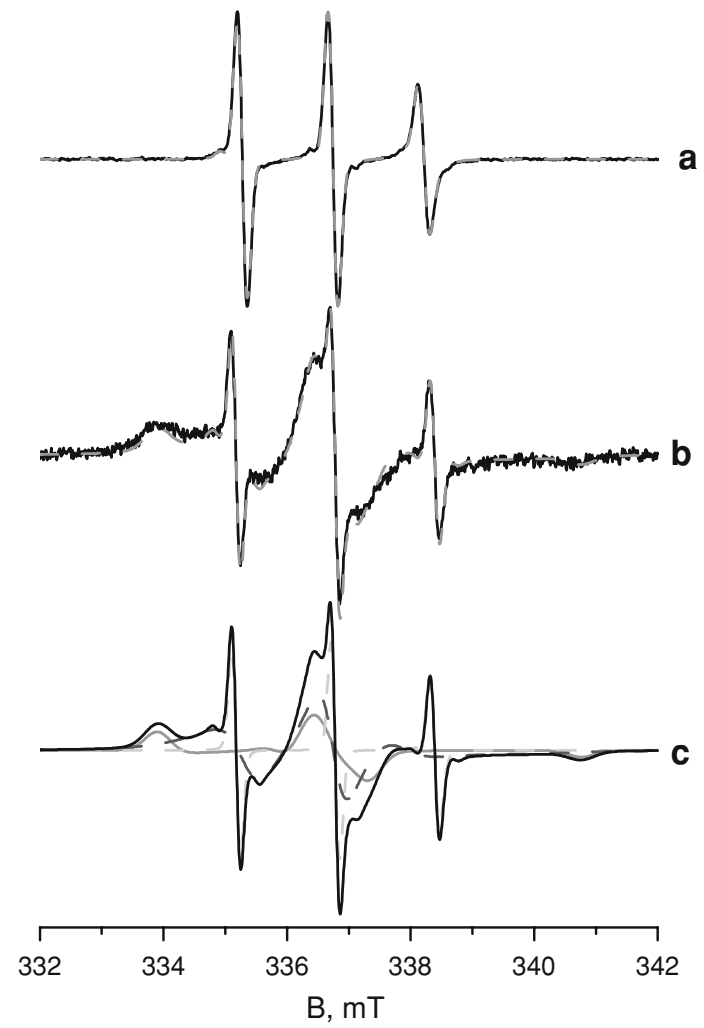

For EPR, samples of SL-A $\beta$ were measured in DMSO to obtain the free, soluble state of $\mathrm{A} \beta$ (Fig. 2a). The spectrum consists of three lines, which are due to the hyperfine interaction (HFI) of the unpaired electron spin of the nitroxide group of the MTSL with the $N$ nucleus $(I=1)$. The small line width indicates a mobile spin label. The simulation (Fig. 2a, dotted line) yields a rotation correlation time $\tau_{\mathrm{r}}$ of 0.26 ns. In Fig. 2 b, the spectrum of a sample containing $40 \%$ of SL-A $\beta$ mixed with $60 \% \mathrm{~A} \beta$ in PBS buffer after 5 min of incubation is shown. The lines are broadened relative to those of SL-A $\beta$ in DMSO, and additional lines are observed, suggesting a superposition of different spectra. This is illustrated in Fig. 2c, where the three components that are needed to simulate the spectrum are shown. These components are subsequently referred to as fast, medium, and slow component and the spectral simulation parameters are given in Table 1.

In Fig. 3, the spectrum of a sample containing $40 \%$ of SL-A $\beta$ mixed with $60 \%$ $\mathrm{A} \beta$ is shown for two selected time points. The spectra can be simulated well (see Fig. 3b) with the parameters given in Table 1. Spin concentration determination (see "Materials and Methods") revealed that the sample accounts for approx. $60 \%$ of the total spins. A sample containing $14 \%$ of SL-A $\beta$ had essentially the same spectra as those containing $40 \%$ SL-A $\beta$, confirming that the line shape of the spectra of these samples is not affected by spin-spin interaction (see below). To test the 
Table 1 EPR parameters of $40 \%$ SL-A $\beta$ in PBS at room temperature for different incubation times (see text). $\tau_{\mathrm{R}}$ is the rotation correlation time, $A_{\mathrm{zz}}$ is the hyperfine splitting along the $z$-direction, $l w$ is the component line width of the simulation and \% stands for percentage of the given component to the total spectrum

\begin{tabular}{|c|c|c|c|c|c|c|c|c|c|c|c|}
\hline & \multicolumn{4}{|l|}{ Fast } & \multicolumn{4}{|c|}{ Medium } & \multicolumn{3}{|l|}{ Slow } \\
\hline & $\begin{array}{l}\tau_{\mathrm{R}} \\
(\mathrm{ns})\end{array}$ & $\begin{array}{l}A_{\mathrm{zz}} \\
(\mathrm{MHz})\end{array}$ & $\begin{array}{l}l w \\
(\mathrm{MHz})\end{array}$ & $\%$ & $\begin{array}{l}\tau_{\mathrm{R}} \\
\text { (ns) }\end{array}$ & $\begin{array}{l}A_{\mathrm{zz}} \\
(\mathrm{MHz})\end{array}$ & $\begin{array}{l}l w \\
(\mathrm{MHz})\end{array}$ & $\%$ & $\begin{array}{l}A_{\mathrm{zz}} \\
(\mathrm{MHz})\end{array}$ & $\begin{array}{l}l w \\
(\mathrm{MHz})\end{array}$ & $\%$ \\
\hline $10 \mathrm{~min}$ & 0.1 & 109.5 & 0.125 & 5.5 & 2.9 & 109.5 & 0.125 & 58 & 96 & 0.49 & 36.5 \\
\hline $5-6 \mathrm{~h}$ & 0.17 & 109.3 & 0.125 & 14 & 2.3 & 109.3 & 0.125 & 52 & 96 & 0.49 & 34 \\
\hline 25 days & 0.12 & 109.3 & 0.125 & 9.5 & 2.3 & 109.3 & 0.125 & 53.5 & 95 & 0.49 & 37.5 \\
\hline Error & \pm 0.03 & \pm 0.2 & \pm 0.005 & \pm 1 & \pm 0.3 & \pm 0.2 & \pm 0.005 & \pm 1 & \pm 0.5 & \pm 0.01 & \pm 1 \\
\hline
\end{tabular}

Fig. 3 Room-temperature EPR spectra of $40 \%$ SL-A $\beta$ in PBS after $5 \mathrm{~h}$ (solid line) and after 25 days (dotted line) (a). Spectrum of $5 \mathrm{~h}$ incubation (solid line) and simulation (dotted line) (b)

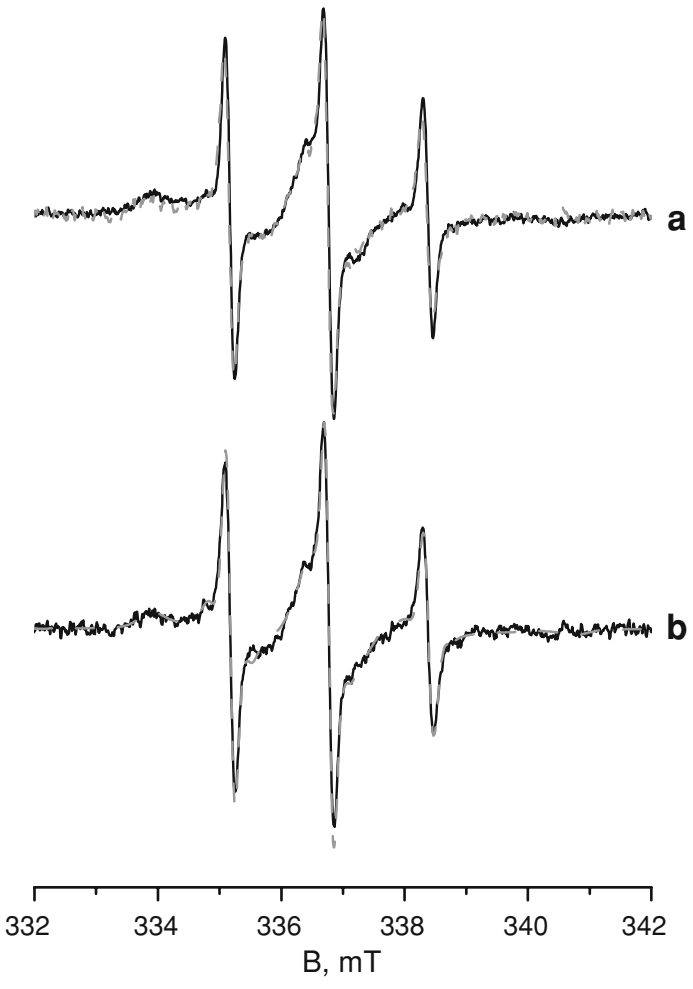

influence of the aggregation conditions, spectra of samples kept still in the measurement capillaries for 9 days were compared to those of a fresh sample drawn from the original incubation solution that was shaken for the same amount of time. In all cases, the spectra were identical, revealing that there is no influence of the sample container (measurement tube or glass vial—see "Materials and Methods") or agitation of the sample (aggregation under resting conditions or during shaking). 
Table 2 Results of filtration experiment. The EPR parameters of the spectra of the filtrate and the retentate of the filtered solution and of the unfiltered solution of the $40 \%$ SL-A $\beta$ in PBS are given (see text). $\tau_{\mathrm{R}}$ is the rotation correlation time, $A_{\mathrm{zz}}$ is the hyperfine splitting along the $z$-direction, $l w$ is the component line width of the simulation and \% stands for percentage of the given component to the total spectrum

\begin{tabular}{|c|c|c|c|c|c|c|c|c|c|c|c|}
\hline & \multicolumn{4}{|l|}{ Fast } & \multicolumn{4}{|c|}{ Medium } & \multicolumn{3}{|l|}{ Slow } \\
\hline & $\begin{array}{l}\tau_{\mathrm{R}} \\
(\mathrm{ns})\end{array}$ & $\begin{array}{l}A_{\mathrm{zz}} \\
(\mathrm{MHz})\end{array}$ & $\begin{array}{l}l w \\
(\mathrm{MHz})\end{array}$ & $\%$ & $\begin{array}{l}\tau_{\mathrm{R}} \\
(\mathrm{ns})\end{array}$ & $\begin{array}{l}A_{\mathrm{zz}} \\
(\mathrm{MHz})\end{array}$ & $\begin{array}{l}l w \\
(\mathrm{MHz})\end{array}$ & $\%$ & $\begin{array}{l}A_{\mathrm{zz}} \\
(\mathrm{MHz})\end{array}$ & $\begin{array}{l}l w \\
(\mathrm{MHz})\end{array}$ & $\%$ \\
\hline Solution & 0.21 & 109.75 & 0.13 & 18 & 2.3 & 109.75 & 0.13 & 51 & 96 & 0.49 & 31 \\
\hline Retentate & 0.31 & 109.75 & 0.13 & 13 & 2.7 & 109.75 & 0.13 & 53 & 96 & 0.49 & 34 \\
\hline Filtrate & 0.17 & 109.75 & 0.13 & 24 & 2.3 & 109.75 & 0.13 & 45.5 & 95 & 0.49 & 30.5 \\
\hline Error & \pm 0.03 & \pm 0.2 & \pm 0.005 & \pm 1 & \pm 0.3 & \pm 0.2 & \pm 0.005 & \pm 1 & \pm 0.5 & \pm 0.01 & \pm 1 \\
\hline
\end{tabular}

\subsection{Filtration Experiment}

A possible interconversion of species found in the mixture can be checked by filtration of the solution with a membrane filter with the suitable molecular weight (MW) cutoff. Here, a sample of $40 \%$ SL-A $\beta$ was filtered through a $30 \mathrm{kDa}$ MWcutoff filter (see "Materials and Methods"). Spectra of the filtrate, the retentate and the unfiltered solution of the sample that was subjected to the filtration procedure were measured (see "Materials and Methods"). A simulation using the parameters in Table 2 gave good agreement with the data. The retentate contains a significant contribution of the fast component, and the filtrate has a composition that is similar to that of the sample that was not filtrated. As larger aggregates cannot pass the filter (nominally, a cutoff of $30 \mathrm{kDa}$ corresponds to a heptamer of $\mathrm{A} \beta$ ), the amount of intermediate and slow fractions should be greatly reduced in the filtrate, and no fast component is expected in the retentate. This suggests that the species responsible for these fractions can interconvert on the time scale of the filtration experiment, in this case of several hours.

\subsection{Frozen-Solution EPR}

Frozen-solution EPR was performed on samples containing pure SL-A $\beta$ in DMSO and in PBS buffer with an incubation time of $5 \mathrm{~min}$ before freezing. The superposition of the two spectra (Fig. 4a) shows that $A_{\mathrm{zz}}$ of SL-A $\beta$ in DMSO is smaller than in PBS and that the spectrum in PBS has some line broadening. Different $A_{\mathrm{zz}}$ values indicate polarity differences in the environment of the spin label [28]. The smaller $A_{\mathrm{zz}}$ of SL-A $\beta$ in DMSO reflects the lower polarity of DMSO relative to the aqueous buffer. The spectrum in DMSO is well simulated with a single species, whereas the spectrum in PBS needs to be simulated as a superposition of two species, one with a component line width identical to that in DMSO, the second with a broader component line width (see Table 3). Under the conditions of the experiment, the broadening can only be due to spin-spin interaction. The distance between the spins is estimated from the comparison of the 
Fig. 4 Frozen-solution EPR spectrum of SL-A $\beta$ in DMSO and PBS (a). Simulation (dotted line) of the spectrum in PBS (solid line) (b)

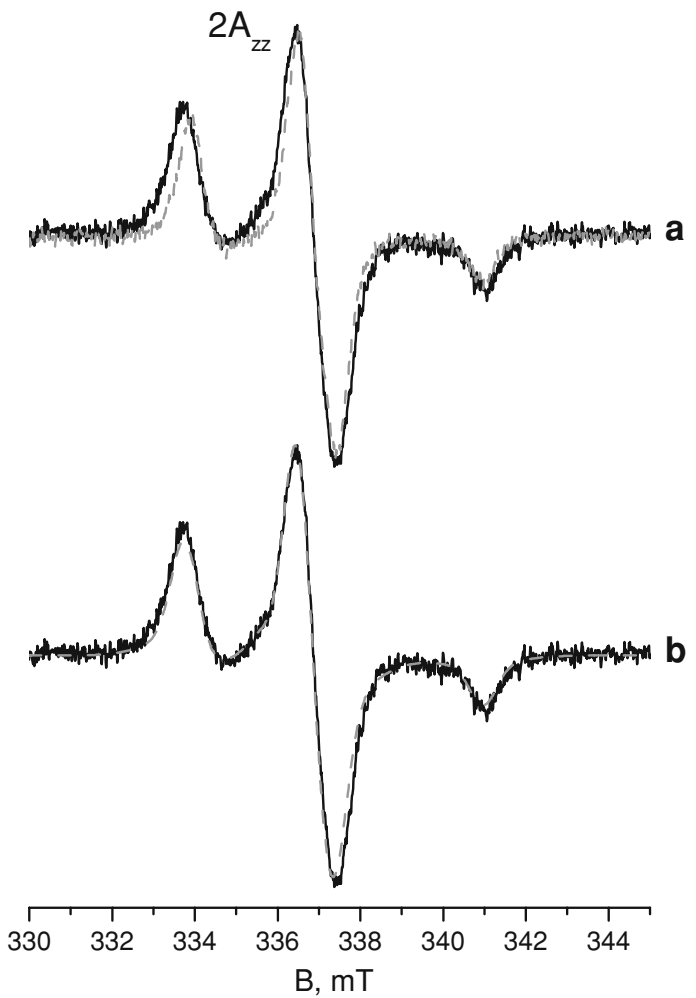

Table 3 Parameters of the frozen-solution EPR spectrum of $100 \%$ SL-A $\beta$ in DMSO and PBS. $A_{\mathrm{zz}}$ is the hyperfine splitting along the $z$-direction, $l w$ is the component line width of the simulation

\begin{tabular}{lccccc}
\hline & Narrow & & & Broad & \\
\cline { 2 - 3 } \cline { 5 - 6 } & $A_{\mathrm{zz}}(\mathrm{MHz})$ & $l w(\mathrm{MHz})$ & & $A_{\mathrm{zz}}(\mathrm{MHz})$ & $l w(\mathrm{MHz})$ \\
\hline DMSO & $98 \pm 0.5$ & $0.75 \pm 0.02$ & & n.a. & n.a. \\
PBS & $101 \pm 1$ & $0.75 \pm 0.02$ & & $101 \pm 8$ & $1.9 \pm 0.3^{\mathrm{a}}$ \\
\hline
\end{tabular}

\footnotetext{
a The percentage of the broadened component is $36 \pm 3 \%$
} n.a. not applicable

second moment [29] of the broadened component with the narrower component of the spectra. For a pair of interacting spins this results in a distance of $1.2 \mathrm{~nm}$.

\subsection{Interpretation of the Rotation Correlation Times of the Fractions}

Molecular volumes corresponding to the fast and intermediate fractions are calculated from the $\tau_{\mathrm{r}}$ values in "Materials and Methods". For the fast fraction, a volume of $606 \AA^{3}$ results, which is close to the volume of $529 \AA^{3}$ obtained from the $\tau_{\mathrm{R}}$ of SL-A $\beta$ in DMSO. This is about half of the calculated volume of $\mathrm{A} \beta$ that is obtained from the molecular weight of $\mathrm{A} \beta$ and assuming a density of $1.52 \mathrm{~g} / \mathrm{cm}^{3}$ as 
suggested in Fischer et al. [30]. The smaller volume determined experimentally is most likely due to the mobility of the spin label, which shortens $\tau_{\mathrm{R}}$. On the other hand, the viscosity of the solution of $\mathrm{A} \beta$ in PBS is certainly larger than that of pure water used in the calculation; thus in this respect the calculated molecular volume is an upper limit.

The volume of $9,304 \AA^{3}$ for the fraction with the intermediate $\tau_{R}$ indicates a multimer of $\mathrm{A} \beta$. To estimate the number of $\mathrm{A} \beta$ peptides in such an aggregate, the molecular volume can be taken (i) from the monomer volume deduced from the fast fraction, or (ii) from the volume of $\mathrm{A} \beta$ expected from the molecular weight. In the first case, a 15-mer results. This number derives from the ratio of $\tau_{\mathrm{R}}$ values measured in the same sample, so the absolute viscosity does not play a role. It assumes, however, that the local mobility of the spin label in the aggregate is the same as in the monomer. Most likely, the mobility of the spin label in the aggregate is lower than in the monomer, suggesting that the true number of molecules in the aggregate is smaller than 15. In the second case, the number of molecules in the aggregate is eight. In that case, the higher viscosity of the $\mathrm{A} \beta$-PBS solution relative to water is not taken into account, which, for the same reasons as discussed above for the monomer, would lead to an underestimate of the number of molecules in the aggregate. We, therefore, place the lower and upper limits of the number of molecules in aggregates of the intermediate fraction at 8 and 15 molecules, respectively.

\section{Discussion}

We have spin-labeled the Cys-A $\beta$ variant with the MTSL label (SL-A $\beta$ ) and have shown by the Congo-red assay and electron microscopy that SL-A $\beta$ and its mixtures with $\mathrm{A} \beta$ form the same type of fibrils as the pure $\mathrm{A} \beta$ under conditions as close as possible to those of the EPR experiments. Fibrils are observed for all these samples, even at the earliest time points tested, and fibril growth is observed for the ensuing hours. The fibrilization occurs within minutes and over a time scale of hours the fibrils grow.

A large body of literature concerns differences in aggregation kinetics and fibril morphology that can be induced by changing aggregation conditions (see, for example, refs. [17-21]). In the present exploratory study, we chose one set of conditions that are reproducible and yield fibrils under the conditions of the EPR experiment.

In EPR, the difference between the spectra in DMSO and PBS gives clear evidence of aggregation. In the frozen solution spectra, line broadening can only be due to the spin-spin interaction. Consequently, in the fraction of the sample with the broadened component, the distance between spin labels is closer than what would be expected for monomers randomly distributed in solution, suggesting aggregation. For this fraction, a distance of $1.2 \mathrm{~nm}$ results for a spin pair.

For the $\mathrm{N}$-terminal region of the $\mathrm{A} \beta$, distances in a lateral direction, i.e., perpendicular to the fibril axis are around $4 \mathrm{~nm}[4,31]$, whereas within the $\beta$-sheet core of the fibrils, intra-strand distances, i.e., distances in the direction along the fibril axis of $0.4 \mathrm{~nm}$ are expected (Protein Data Bank entry, 2beg.pdb [31]). Due to 
the $1 / r^{3}$ dependence of the dipolar interaction, lateral distances are so large that they should not contribute significantly to the broadening. The intra-strand distance, on the other hand, should result in a larger broadening than experimentally observed. Furthermore, in this direction, interactions with multiple partners are expected, causing even larger broadening than that of a pair interaction. In our construct, however, the spin label is in the $\mathrm{N}$-terminal region of $\mathrm{A} \beta$, i.e., outside the $\beta$-sheet region of the fibrils. Spin-label EPR on fully developed fibrils has shown that spin labels in the N-terminal region of $\mathrm{A} \beta[8]$ and of other fibril forming proteins [9] are less affected by fibrilization than spin labels in the central regions of the $\beta$ sheet. We, therefore, attribute the smaller broadening to an overall larger distance between spin labels because of a distribution of conformations within the N-terminal region of $\mathrm{A} \beta$ that also diminishes second-neighbor interactions.

The broadening of the room-temperature spectra reveals a reduced mobility of the spin labels. Aggregation can reduce the mobility of the spin label, if the spin label becomes trapped within the aggregates, and if the aggregates are sufficiently large to be rotating slowly on the EPR time scale.

The room temperature EPR spectra of the peptides in PBS revealed three components, corresponding to fractions of the samples in which the spin labels have different mobility. The rotation-correlation time $\tau_{R}$ of the fastest component is attributed to the monomeric $\mathrm{A} \beta$. The medium component corresponds to an oligomer with a minimum number of 8 and a maximum number of 15 molecules and the slow one to larger aggregates that are immobilized on the EPR time scale (see "Results").

The filtration experiment shows that monomers must be formed from the larger aggregates that do not pass the membrane filter (monomer-like SL-A $\beta$ in the retentate) and that larger aggregates are formed from the smaller aggregates passing the $30 \mathrm{kDa}$ filter, suggesting interconversion between species on the time scale of hours.

With respect to the time course of aggregation, we find evidence of aggregation at the earliest times. The frozen solution experiment reflects a time point of $5 \mathrm{~min}$ (see "Filtration Experiment"), and, in the room temperature experiment, the broadened components are evident from the earliest time points (see Figs. 2, 3; Table 1). The composition of the spectra corresponding to different time points reveals percentage changes of the components that are significant given the error of the simulation parameters. For the present set of experiments, however, the differences are close to the sample-to-sample variation, so at present we disregard the temporal changes of these components.

This investigation shows that spectral signatures of aggregation can be obtained by cw EPR and that, in principle at least, information on aggregate size and the time development thereof could become accessible. We show that the method should be sensitive enough to unravel oligomers of a wide variety of sizes and thus would be able to detect the time development of oligomers in aggregating samples. Even small oligomers of a couple of $\mathrm{A} \beta$ molecules should be detectable and time resolution down to several minutes can be achieved. In this respect it surpasses the presently most widely used chromatographic techniques. Positioning the spin label closer to the $\beta$-sheet core of $\mathrm{A} \beta$ will reduce the local mobility of the spin label once 
A $\beta$ forms part of an oligomer or aggregate, improving the sensitivity of the spinlabel motion to the properties of the aggregate. The most faithful reporters of the aggregate motion would be spin labels that form part of the peptide backbone, such as the TOAC label $[32,33]$ or other non-natural amino-acid replacement labels [34]. The challenge, however, is to incorporate these spin labels into the $\mathrm{A} \beta$ sequence and to purify the resulting product. Future studies based on the $\mathrm{A} \beta$ constructs mentioned will have a higher sensitivity to the formation of aggregates in the sample. Thereby, a refined picture of the initial stages of aggregation will be obtained, a step towards understanding the fibrillization process.

Acknowledgments Discussion and support by Edgar Groenen are gratefully acknowledged. We thank Maryam Hashemi-Shabestari for careful reading of the manuscript and Gijs van der Marel for support. This work is part of the research programme of the 'Stichting voor Fundamenteel Onderzoek der Materie (FOM)', which is financially supported by the 'Nederlandse Organisatie voor Wetenschappelijk Onderzoek (NWO)', Grant 03BMP03. Financial support from NWO, the Dutch scheince organization Grants 700.50.026 and TOP Grant 700.53.305 are gratefully acknowledged.

Open Access This article is distributed under the terms of the Creative Commons Attribution Noncommercial License which permits any noncommercial use, distribution, and reproduction in any medium, provided the original author(s) and source are credited.

\section{References}

1. C. Sachse, M. Fandrich, N. Grigorieff, Proc. Natl. Acad. Sci. USA 105, 7462-7466 (2008)

2. D. Losic, L.L. Martin, A. Mechler, M.I. Aguilar, D.H. Small, J. Struct. Biol. 155, 104-110 (2006)

3. J.J. Balbach, A.T. Petkova, N.A. Oyler, O.N. Antzutkin, D.J. Gordon, S.C. Meredith, R. Tycko, Biophys. J. 83, 1205-1216 (2002)

4. A.T. Petkova, Y. Ishii, J.J. Balbach, O.N. Antzutkin, R.D. Leapman, F. Delaglio, R. Tycko, Proc. Natl. Acad. Sci. USA 99, 16742-16747 (2002)

5. A.T. Petkova, W.M. Yau, R. Tycko, Biochemistry 45, 498-512 (2006)

6. J. Danielsson, R. Pierattelli, L. Banci, A. Gräslund, FEBS J. 274, 46-59 (2007)

7. M. Margittai, R. Langen, Methods Enzymol. 413, 122-139 (2006)

8. M. Törok, S. Milton, R. Kayed, P. Wu, T. McIntire, C.G. Glabe, R. Langen, J. Biol. Chem. 277, 40810-40815 (2002)

9. M. Chen, M. Margittai, J. Chen, R. Langen, J. Biol. Chem. 282, 24970-24979 (2007)

10. R. Nelson, M.R. Sawaya, M. Balbirnie, A.O. Madsen, C. Riekel, R. Grothe, D. Eisenberg, Nature 435, 773-778 (2005)

11. J. Danielsson, J. Jarvet, P. Damberg, A. Gräslund, Magn. Reson. Chem. 40, S89-S97 (2002)

12. F. Eghiaian, T. Daubenfeld, Y. Quenet, M. van Audenhaege, A.P. Bouin, G. van der Rest, J. Grosclaude, H. Rezaei, Proc. Natl. Acad. Sci USA 104, 7414-7419 (2007)

13. A. Peralvarez-Marin, A. Barth, A. Gräslund, J. Mol. Biol. 379, 589-596 (2008)

14. W. Qi, A. Zhang, D. Patel, S. Lee, J.L. Harrington, L.M. Zhao, D. Schaefer, T.A. Good, E.J. Fernandez, Biotechnol. Bioeng. 100, 1214-1227 (2008)

15. K.M. Lundberg, C.J. Stenland, F.E. Cohen, S.B. Prusiner, G.L. Millhauser, Chem. Biol. 4, 345-355 (1997)

16. A. Peralvarez-Marin, A. Gräslund, Biophys. J. Suppl. S, 42A-42A (2007)

17. S. Lee, E.J. Fernandez, T.A. Good, Protein Sci. 16, 723-732 (2007)

18. A.K. Paravastu, A.T. Petkova, R. Tycko, Biophys. J. 90, 4618-4629 (2006)

19. A.T. Petkova, R.D. Leapman, Z.H. Guo, W.M. Yau, M.P. Mattson, R. Tycko, Science 307, 262-265 (2005)

20. J. McLaurin, D.S. Yang, C.M. Yip, P.E. Fraser, J. Struct. Biol. 130, 259-270 (2000) 
21. L.O. Tjernberg, A. Pramanik, S. Bjorling, P. Thyberg, J. Thyberg, C. Nordstedt, K.D. Berndt, L. Terenius, R. Rigler, Chem. Biol. 6, 53-62 (1999)

22. S. Stoll, A. Schweiger, J. Magn. Reson. 178, 42-55 (2006)

23. S. Steigmiller, M. Börsch, P. Gräber, M. Huber, Biochim. Biophys. Acta Bioenerg. 1708, 143-153 (2005)

24. D.K. Wilkins, C.M. Dobson, M. Gross, Eur. J. Biochem. 267, 2609-2616 (2000)

25. J. Sereikaite, V.A. Bumelis, Acta Biochim. Pol. 53, 87-92 (2006)

26. C.S. Goldsbury, S. Wirtz, S.A. Muller, S. Sunderji, P. Wicki, U. Aebi, P. Frey, J. Struct. Biol. 130, 217-231 (2000)

27. J.D. Harper, C.M. Lieber, P.T. Lansbury, Chem. Biol. 4, 951-959 (1997)

28. R. Owenius, M. Engström, M. Lindgren, M. Huber, J. Phys. Chem. A 105, 10967-10977 (2001)

29. H.J. Steinhoff, Front. Biosci. 7, C97-C110 (2002)

30. H. Fischer, I. Polikarpov, A.F. Craievich, Protein Sci. 13, 2825-2828 (2004)

31. T. Luhrs, C. Ritter, M. Adrian, D. Riek-Loher, B. Bohrmann, H. Doeli, D. Schubert, R. Riek, Proc. Natl. Acad. Sci. USA 102, 17342-17347 (2005)

32. A.D. Milov, Y.D. Tsvetkov, F. Formaggio, M. Crisma, C. Toniolo, J. Raap, J. Am. Chem. Soc. 123, 3784-3789 (2001)

33. A. Bettio, V. Gutewort, A. Poppl, M.C. Dinger, O. Zschornig, K. Arnold, C. Toniolo, A.G. BeckSickinger, J. Pept. Sci. 8, 671-682 (2002)

34. C.F.W. Becker, K. Lausecker, M. Balog, T. Kalai, K. Hideg, H.J. Steinhoff, M. Engelhard, Magn. Reson. Chem. 43, S34-S39 (2005) 